\title{
Avaliação da influência da adição de fibras de aço no concreto fluido solicitado por tensões de tração
}

\author{
Evaluation of steel fibers addition in fluid concrete subjected to tensile stress
}

\author{
F.A. P. Góis ${ }^{1 *}$; A. S. R. Barboza ${ }^{2}$ \\ ${ }^{1}$ Departamento de Arquitetura e Urbanismo/ Universidade Federal de Sergipe, 49170-000, Laranjeiras-Sergipe, \\ Brasil \\ ${ }^{2}$ Centro de Tecnologia, Universidade Federal de Alagoas, 57052-970, Maceió-Alagoas, Brasil
}

*fernanda_gois@hotmail.com

(Recebido em 10 de junho de 2015; aceito em 04 de novembro de 2015)

\begin{abstract}
O desenvolvimento de novas tecnologias para a produção do concreto, ao longo do tempo, vem permitindo elevar a sua resistência à compressão, melhorar a trabalhabilidade e garantir maior durabilidade para as estruturas executadas com o mesmo. Apesar de suas muitas vantagens e melhorias atingidas, o concreto quando aplicado em situações nas quais surgem esforços de tração apresenta limitações relacionadas ao seu comportamento frágil. Uma alternativa para contornar este problema é a utilização de armadura dispersa, por meio de fibras imersas no concreto, obtendo-se assim um material compósito que possui características diferentes do material original. Buscando colaborar na avaliação da eficiência da adição de fibras, o presente trabalho analisa o comportamento de concretos fluidos reforçados com fibras de aço, submetidos a esforços de tração, considerando como parâmetros de influência o fator de forma e a fração volumétrica das fibras. Para a referida avaliação, foram utilizados corpos-de-prova cilíndricos, de dimensões de $10 \mathrm{~cm}$ x $20 \mathrm{~cm}$ de concreto fluido de resistência normal, com adição de fibras de aço nos teores de $0,4 \%$ e $0,8 \%$ da quantidade de cimento em massa e com fatores de forma de 33, 50 e 80 . Os corpos de prova foram submetidos a ensaios de tração por compressão diametral, e foi possível foi possível afirmar com os resultados obtidos, que a matriz cimentícia fluida, com adição de fibras com maior fator de forma e maior fração volumétrica, tende a ser mais eficiente na resposta do concreto submetido a esforços de tração.

Palavras-chave: Concreto Fluido Reforçado com Fibras. Fibras de aço. Tração por Compressão Diametral.
\end{abstract}

The development of new technologies for the production of concrete, over the time, have contributed to increase its compressive strength, improve workability and to ensure durability for structures executed with the same. Despite its many advantages and improvements achieved, the concrete when applied in situations which arise tensile stresses shows limitations related to its brittle behavior. An alternative to overcome this problem is to use dispersed fibers immersed in the concrete, thus creating a composite material with different characteristics from the original material. In order to collaborate in analysing the added fiber efficiency, this paper deals about the behavior of steel fibers reinforced fluid concrete when tensile stresses is applied, considering fiber aspect relation form and volume fraction the influence parameters. For this evaluation, we used cylindrical specimens, dimensions of $10 \mathrm{~cm}$ x $20 \mathrm{~cm}$, considering $0.4 \%$ and $0.8 \%$, related cement weight, to steel fibers addition and relation aspect form of 33 , 50 and 80 . Tensile tests by diametrical compression was done and it was possible to say that steel fiber reinforced fluid concrete is more efficient when tensile stress is applied with larger relation aspect form and greater volumetric fraction, it tends to be more efficient in response concrete subjected to tensile stress.

Keywords: Reinforced Fiber Fluid Concrete. Steel Fibers. Tensile Stress in Diametric Compression.

\section{INTRODUÇÃO}

O concreto se destaca como um dos materiais mais utilizados no setor da construção civil, devido principalmente a sua enorme capacidade de adaptação a diversos tipos de formas e condições de produção. Embora detenha inúmeras vantagens, o concreto é um material que apresenta ruptura frágil e baixa resistência a esforços de tração. 
Uma das alternativas tecnológicas empregadas para melhorar o desempenho do concreto, quando submetido a esforços de tração, consiste na incorporação de barras de aço, imersas na matriz cimentícia, dando origem ao concreto armado. Novos estudos vêm sendo realizados e uma nova alternativa, com o intuito de melhorar as características deste material, surge com a possibilidade de reforço através do uso de armadura dispersa, por meio da incorporação de fibras, à massa de concreto.

O concreto reforçado com fibras é um material compósito formado por uma matriz cimentícia e por certa quantidade de fibras descontínuas. Segundo Figueiredo (2011) [1], devido à ação das fibras, o novo material apresenta um comportamento pseudo-dúctil, ou seja, continua apresentando uma resistência residual a esforços nele aplicados, mesmo após sua fissuração.

De acordo com Mehta e Monteiro (2008) [11], o concreto apresenta resistência à tração bem inferior à resistência à compressão, cuja relação está geralmente em torno de $7 \%$ a $11 \%$. O motivo de tão grande diferença entre as resistências à tração e à compressão é atribuído à heterogênea e complexa microestrutura do concreto, associado à sua grande dificuldade de interromper a propagação das fissuras quando submetidos tensões de tração.

A introdução de fibras, segundo Pujadas (2013) [12], modifica o comportamento não linear do concreto, sobretudo em relação à tração, impedindo, assim, a abertura e propagação de fissuras. Uma vez que o concreto já esteja fissurado, e a ligação da interface fibra/matriz seja suficientemente, as fibras podem descolar da matriz e então formar ligamentos em formato de "ponte" nas fissuras. Esse efeito ponte (bridging effect) das fibras, segundo Pujadas (2013) [12], aumenta a resistência residual do compósito e consequentemente devido à perda de aderência (debonding) e arrancamento (pull-out), dissipa uma maior energia, o que conduz a um importante incremento da tenacidade e um maior controle da fissuração.

Praticamente todos os concretos, sejam plásticos, fluidos ou secos, de baixa ou alta resistência, podem ser reforçados com fibras. No entanto, de acordo com Bentur e Mindess (1990) [8], a trabalhabilidade é afetada pela adição de fibras, em proporção direta à fração volumétrica das fibras utilizadas. Concretos reforçados com altos teores de fibras, produzidos de forma convencional, apresentam difícil dispersão das fibras, podendo-se observar $o$ agrupamento de fibras e consequentemente a formação de novelos.

Sendo assim, a adição de fibras se torna mais eficiente quando incorporada a um concreto fluido, por proporcionar incremento de desempenho no estado endurecido, mas sem comprometer o desempenho no estado fresco.

Neste contexto, este trabalho se propõe a produzir e avaliar o comportamento de um concreto fluido com diferentes teores e geometrias de fibras de aço.

\section{MATERIAL E MÉTODOS}

Nesta seção são descritos os materiais, os equipamentos e o procedimento experimental para a determinação da influência das características físicas (fator de forma e fração volumétrica) das fibras de aço, nos concretos fluidos produzidos. Além disso, a definição da dosagem é apresentada.

Foram adotados como amostras para os ensaios, corpos de prova cilíndricos de dimensões 10 $\mathrm{cm}$ x $20 \mathrm{~cm}$, e os mesmos foram solicitados por compressão diametral, aos 28 dias de idade. Os corpos de prova foram produzidos e ensaiados no Laboratório de Estruturas e Materiais (LEMA) do Núcleo de Pesquisas Tecnológicas (NPT) da Universidade Federal de Alagoas (UFAL).

Foram produzidas 07 dosagens diferentes para o concreto, nas quais, para viabilizar comparações entre as mesmas, mantiveram-se constantes todos os componentes da matriz cimentícia, com exceção da fração volumétrica e do fator de forma das fibras. Os concretos estudados foram referenciados com a seguinte nomenclatura: um concreto de referência sem fibras (CREF-S/F) e seis concretos reforçados com fibras de aço (CRFA). 


\subsection{MATERIAL}

O cimento utilizado foi do tipo Portland Composto CP II-F-32, de um mesmo lote de fabricação. As características físicas e químicas do mesmo foram fornecidas pelo fabricante, e se enquadram nos requisitos da norma NBR 11578 (ABNT, 1997) [6].

O agregado graúdo utilizado foi brita de origem granítica com dimensão máxima característica de $12,5 \mathrm{~mm}$. Somente um tipo de areia foi usado para a produção de todos os concretos desta pesquisa, classificada como areia média, de acordo com a norma NBR 7211 (ABNT, 2005) [4] cuja dimensão máxima característica foi de 2,4 mm.

$\mathrm{Na}$ Tabela 1, são apresentadas as características da areia e da brita utilizadas e, na Figura 1, são apresentadas suas respectivas curvas granulométricas.

Tabela 1: Caracterização dos Agregados.

\begin{tabular}{ccc}
\hline Caracterização & Areia Média & Brita 12,5 mm \\
\hline Massa Específica $\left(\mathbf{g} / \mathbf{c m}^{\mathbf{3}}\right)$ & 2,611 & 2,685 \\
Massa Unitária do Estado Solto $\left(\mathbf{g} / \mathbf{c m}^{\mathbf{3}}\right)$ & 1,482 & 1,385 \\
Massa Unitária do Estado Compactado $\left(\mathbf{g} / \mathbf{c m}^{\mathbf{3}}\right)$ & - & 1,508 \\
Módulo de Finura & 2,424 & - \\
Absorção $(\%)$ & 0,644 & 0,625 \\
\hline
\end{tabular}

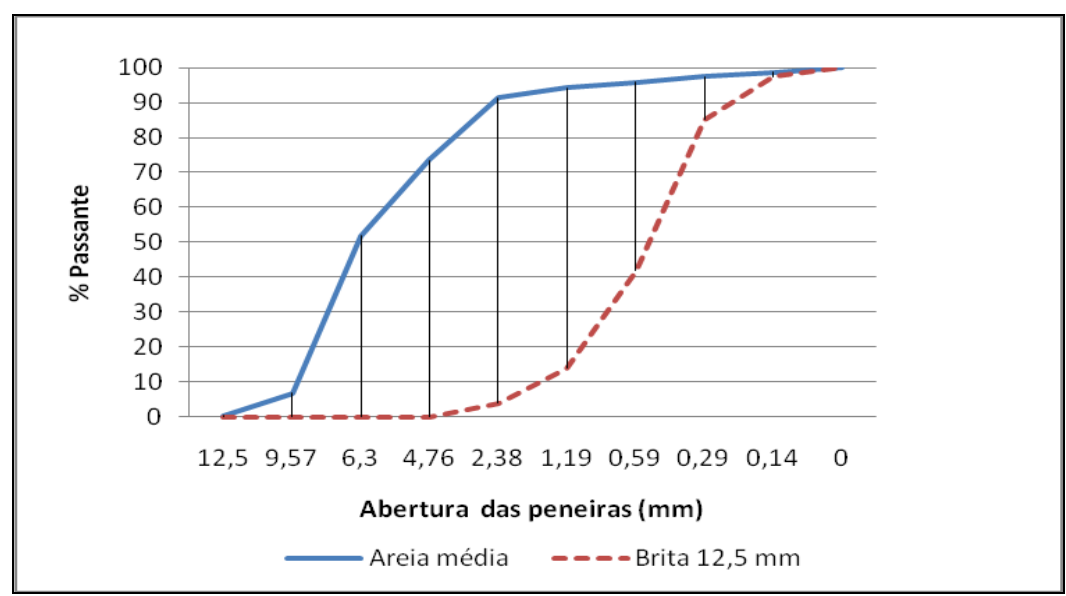

Figura 1 - Curvas Granulométricas dos Agregados.

Para não promover alteração na relação água/cimento, foi utilizado, neste estudo, o plastificante Centrament N3.

Foram utilizadas fibras de aço com ancoragem em gancho nas extremidades, obtidas através do processo de trefilação a frio do aço, com resistência à tração em torno de $1000 \mathrm{MPa}$. Conforme classificação da norma NBR 15530 (ABNT, 2007) [7], a fibra de aço usada neste estudo corresponde ao tipo denominado AI.

Foram consideradas como variáveis de estudo, o fator de forma das fibras (relação comprimento/diâmetro) e o teor de fibras incorporadas no compósito.

Para a variação do fator de forma foram utilizados três tipos de fibras:

a) as fibras curtas que apresentam comprimento (l) de $25 \mathrm{~mm}$ e seção transversal circular com diâmetro (d) de $0,75 \mathrm{~mm}$, resultando num fator de forma de 33, conforme Figura 2 (a);

b) as fibras médias que apresentam comprimento (l) de $30 \mathrm{~mm}$ e seção transversal circular com diâmetro (d) de 0,60 mm, resultando num fator de forma de 50, conforme Figura 2 (b);

c) as fibras longas apresentam comprimento (l) de $60 \mathrm{~mm}$ e seção transversal circular com diâmetro (d) de 0,75 mm, resultando um fator de forma de 80, conforme Figura 2 (c). 

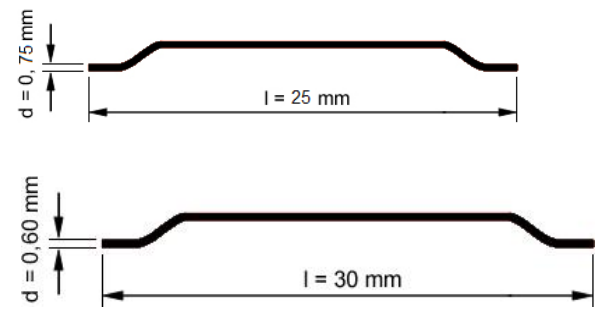

(a)

(b)

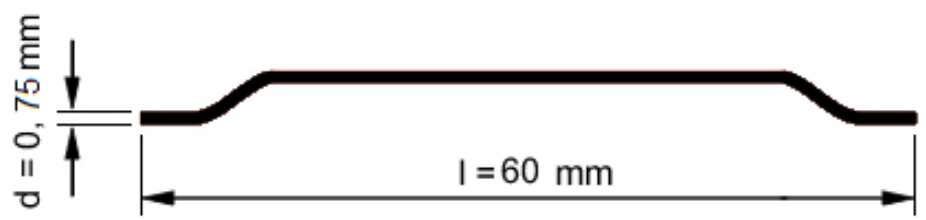

(c)

Figura 1 - Fibras de aço utilizadas no estudo: (a) curta; (b) média; (c) longa.

Para a variação do teor de fibras, foram estipuladas duas frações volumétricas, correspondendo a $0,4 \%$ e $0,8 \%$.

Por fim, para a produção dos concretos foi utilizada água potável fornecida pela Companhia de Saneamento de Alagoas.

As modificações na dosagem do concreto aconteceram apenas pelas mudanças na fração volumétrica e no fator de forma das fibras, sendo as mesmas listadas na Tabela 2. O traço do concreto da pesquisa não foi modificado com a adição de fibras, apenas acrescentaram-se as fibras de aço nas quantidades descritas na Tabela 2.

Tabela 2 - Dosagens dos concretos CREF-S/F e CRFA.

\begin{tabular}{|c|c|c|c|c|c|}
\hline \multirow{2}{*}{ Concreto } & \multirow{2}{*}{$\begin{array}{c}\text { Cimento } \\
{\left[\mathrm{kg} / \mathrm{m}^{3}\right]}\end{array}$} & \multirow{2}{*}{$\begin{array}{c}\text { Água } \\
{\left[\mathrm{kg} / \mathrm{m}^{3}\right]}\end{array}$} & \multicolumn{2}{|c|}{ Agregados [kg/m³] } & \multirow{2}{*}{$\begin{array}{c}\text { Fibras } \\
{\left[\mathrm{kg} / \mathrm{m}^{3}\right]}\end{array}$} \\
\hline & & & Areia & Brita & \\
\hline CREF-S/F & 400 & 200 & 840,72 & 942,5 & 0 \\
\hline CRFA-C1 & 400 & 200 & 840,72 & 942,5 & 31,52 \\
\hline CRFA-C2 & 400 & 200 & 840,72 & 942,5 & 63,04 \\
\hline CRFA-M1 & 400 & 200 & 840,72 & 942,5 & 31,52 \\
\hline CRFA-M2 & 400 & 200 & 840,72 & 942,5 & 63,04 \\
\hline CRFA-L1 & 400 & 200 & 840,72 & 942,5 & 31,52 \\
\hline CRFA-L2 & 400 & 200 & 840,72 & 942,5 & 63,04 \\
\hline
\end{tabular}

Onde:

CREF-S/F: Concreto de referência sem fibras;

CRFA-C1: Concreto reforçado com teor de $0,4 \%$ de fibras curtas de aço;

CRFA-C2: Concreto reforçado com teor de $0,8 \%$ de fibras curtas de aço;

CRFA-M1: Concreto reforçado com teor de $0,4 \%$ de fibras médias de aço;

CRFA-M2: Concreto reforçado com teor de $0,8 \%$ de fibras médias de aço;

CRFA-L1: Concreto reforçado com teor de $0,4 \%$ de fibras longas de aço;

CRFA-L2: Concreto reforçado com teor de $0,8 \%$ de fibras longas de aço. 


\subsection{MÉTODOS DE ENSAIOS}

\subsubsection{ESTADO PLÁSTICO}

Para avaliação dos compósitos no estado plástico foi realizado ensaio de abatimento do tronco de cone, segundo a norma NBR NM 67 (ABNT,1998) [2].

A moldagem dos corpos-de-prova foi realizada com adensamento externo, por mesa vibratória. Terminada a moldagem, os corpos-de-prova eram mantidos em temperatura ambiente por um tempo de 24 horas, até serem desmoldados. Feita a desmoldagem, os corpos-de-prova eram levados à cura em tanque de imersão, onde permaneciam submersos até a data de ensaio, aos 28 dias.

\subsubsection{ESTADO ENDURECIDO}

Para a avaliação dos compósitos no estado endurecido foram realizados ensaios de resistência à compressão, seguindo as recomendações da norma NBR 5739 (ABNT, 2007) [3]; e resistência à tração por compressão diametral, conforme as recomendações da norma NBR 7222 (ABNT, 1994) [5]. Para cada dosagem eram produzidas seis amostras, sendo três delas cada tipo de ensaio. A Figura 3 mostra a execução desses ensaios.

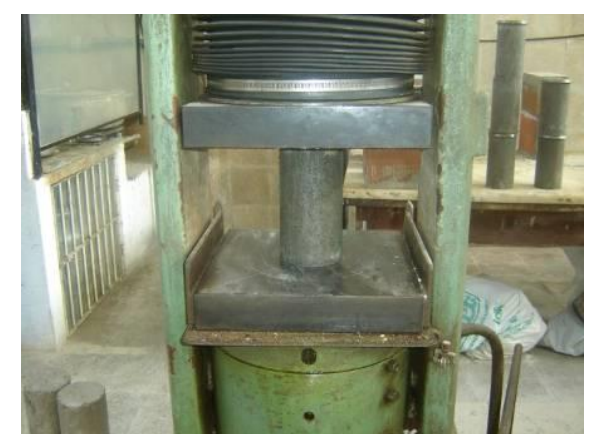

(a)

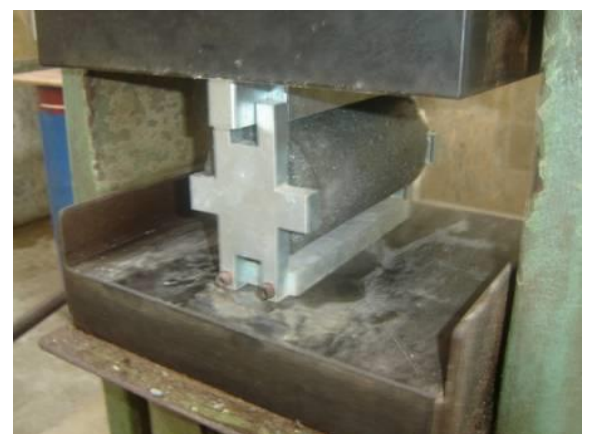

(b)

Figura 3 - Execução dos ensaios: (a) resistência à compressão; (b) resistência à tração por compressão diametral.

\section{RESULTADOS E DISCUSSÃO}

\subsection{PROPRIEDADE REOLÓGICA}

A Tabela 3 apresenta os resultados obtidos com o ensaio do abatimento do tronco de cone executados de acordo com a norma NBR NM 67 (ABNT, 1998) [2].

Tabela 3 - Resultado do ensaio de abatimento do tronco de cone.

\begin{tabular}{cc}
\hline Concreto & Abatimento (cm) \\
\hline CREF-S/F & 20 \\
CRFA-C1 & 19 \\
CRFA-C2 & 17 \\
CRFA-M1 & 19 \\
CRFA-M2 & 15 \\
CRFA-L1 & 13 \\
CRFA-L2 & 12 \\
\hline
\end{tabular}


Conforme os resultados apresentados na Tabela 3, todos os concretos apresentaram consistência adequada sob o seu peso próprio. Estes valores encontram-se na faixa de valores característicos de concretos fluidos e moles, conforme a classificação definida por Aldolfato (2002) [1] e apresentada na Tabela 4. Um concreto mole é considerado um concreto com boa trabalhabilidade e um concreto fluido é considerado um concreto com ótima trabalhabilidade. No primeiro pode ser necessário adensamento mecânico e no segundo pode ser dispensado o adensamento mecânico.

\begin{tabular}{cc} 
Tabela 4 - Classificação das consistências dos concretos. \\
\cline { 2 - 2 } Consistência & Abatimento (cm) \\
\hline Seca & 0 a 2 \\
Firme & 2 a 5 \\
Média & 5 a 12 \\
Mole & 12 a 18 \\
Fluida & 18 a 25 \\
\hline
\end{tabular}

A adição de fibras causou a diminuição do abatimento, tomando-se como referência a mistura CREF-S/F. Durante a realização do ensaio, observou-se que as misturas apresentaram boa retenção de água, sem exsudação e homogeneidade, com todos os constituintes, inclusive as fibras, permanecendo uniformemente distribuídos em toda a matriz cimentícia.

Os resultados do abatimento mostraram que a adição de um teor de $0,4 \%$ de fibras apresentou uma redução de trabalhabilidade idêntica para um fator de forma de 33 e de 50, igual a 5,3\%, já para as fibras com fator de forma de 80 , chegou-se a uma redução significativa de 53,8\%. Para os concretos com teores de $0,8 \%$, observou-se uma redução gradual à medida que o fator de forma aumentava com valores de $17,6 \%, 33,3 \%$ e $66,7 \%$, respectivamente para os fatores de forma de 33,50 e 80.

\subsection{COMPORTAMENTO MECÂNICO}

Os valores médios, excluídos aqueles de maior dispersão, obtidos para os ensaios de resistência à compressão e resistência à tração por compressão diametral podem ser observados na Tabela 5.

Tabela 5 - Propriedades mecânicas dos concretos estudados, aos 28 dias.

\begin{tabular}{ccc}
\hline & \multicolumn{2}{c}{ Resistência dos Concretos (MPa) } \\
\cline { 2 - 3 } Concreto & Compressão & Tração Indireta \\
\hline CREF-S/F & 19,41 & 2,63 \\
CRFA-C1 & 23,71 & 2,75 \\
CRFA-C2 & 23,15 & 2,66 \\
CRFA-M1 & 22,91 & 2,75 \\
CRFA-M2 & 21,76 & 3,05 \\
CRFA-L1 & 21,83 & 3,06 \\
CRFA-L2 & 24,45 & 3,69 \\
\hline
\end{tabular}

Os resultados médios obtidos para as resistências à compressão e à tração indireta estiveram dentro do previsto para as resistências características estimadas e os mesmos demonstraram que o concreto com o maior teor de fibras e o maior fator de forma apresentou melhor desempenho nestas duas propriedades, em comparação com as outras misturas.

O mesmo estudo comprovou, experimentalmente, a influência nos valores de resistência à compressão e à tração do compósito, com a variação do teor da fibra e do fator de forma. Observou-se nitidamente que há um ganho de resistências com o incremento das fibras de aço, quando comparados com a matriz cimentícia de referência sem reforço de fibras de aço. 
Sabe-se que uma das principais limitações do concreto é sua baixa resistência à tração, que é de apenas 7 a $11 \%$ de sua resistência à compressão em concretos usuais. Para os concretos produzidos pelo presente estudo, foi possível observar que a adição de fibras de fator de forma igual a 33 e com teores de $0,4 \%$ e $0,8 \%$ de fibras resultou numa resistência à tração indireta em torno de $11,7 \%$ da resistência à compressão. Para os concretos com fibras com fator de forma de 50, a resistência à tração indireta foi em média $13,1 \%$ da resistência à compressão. Já nos concretos com fibras com fator de forma de 80 , observou-se que a resistência à tração foi em torno de $14,5 \%$ em relação à resistência à compressão.

Para todas as dosagens com adição de fibras, a relação entre a resistência à tração e a resistência à compressão foi superior a $10 \%$. A resistência à tração por compressão diametral apresentou um significativo aumento em relação à matriz de referência sem a adição de fibras, em todos os concretos estudados.

Observa-se na Tabela 5, que nos concretos CRFA-C1, CRFA-M1 e CRFA-L1, com teor igual a $0,4 \%$ e diferentes fatores de forma de fibras, ocorreu um incremento na resistência à tração igual a 4,6\%, nos fatores de forma de 33 e 50, e um aumento de cerca de 16,4\% no concreto com o maior fator de forma. Nos demais concretos, CRFA-C2, CRFA-M2 e CRFA-L2, alterando-se o volume de fibras para o dobro, observou-se um incremento de $1,2 \%, 16 \%$ e $40,3 \%$, respectivamente, nos fatores de 33,50 e 80 , em relação ao concreto de referência sem fibras.

\section{CONCLUSÃO}

O estudo demostrou que, para a variação incremental do teor de fibras e do aumento do fator de forma, o ensaio de abatimento do tronco de cone foi adequado para a medida da consistência de concretos reforçados com $0,4 \%$ e $0,8 \%$ de fibras, de modo que se estabeleça uma quantidade de $63,04 \mathrm{~kg} / \mathrm{m}^{3}$. Notou-se, ainda, que os teores adicionados mantiveram o concreto enquadrado na faixa de valores característicos de concretos fluidos e moles, ou seja, que podem dispensar ou eliminar o adensamento mecânico.

No estudo experimental, demonstrou-se que os compósitos reforçados com fibras longas (fator de forma de 80), cujo comprimento é quatro vezes o diâmetro máximo do agregado, apresentaram maior eficiência tanto na resistência à compressão, quanto na tração indireta, e que, quando são usadas fibras de mesma fração volumétrica, o fator de forma mais elevado tende a apresentar melhores resultados.

A adição de fibras de aço ao concreto proporcionou um aumento médio de 17,9\% para a resistência à compressão e, na resistência à tração indireta do concreto, um aumento de 19,2\% nos concretos com $0,8 \%$ de fibras de aço.

Medidas que garantem trabalhabilidade devem ser tomadas quando se planeja a incorporação de fibras de aço, especialmente em grandes teores e altos fatores de forma, havendo a necessidade do uso de aditivo plastificante para melhorar essa trabalhabilidade perdida na adição das fibras.

\section{AGRADECIMENTOS}

Os autores agradecem especialmente ao Conselho Nacional de Desenvolvimento Científico e Tecnológico (CNPq), ao Laboratório de Estruturas e Materiais (LEMA) do Núcleo de Pesquisas Tecnológicas (NPT) da Universidade Federal de Alagoas (UFAL), bem como aos funcionários pela sua presteza e colaboração. Agradece também às empresas MC-Bauchemie pela doação dos aditivos e à Steel Fibras pela doação das fibras de aço. 


\section{REFERÊNCIAS BIBLIOGRÁFICAS}

1. Andolfato RP. Controle tecnológico básico do concreto [internet]. Ilha Solteira (SP): Universidade Estadual Paulista; 2002. Disponível em:

http://www.nepae.feis.unesp.br/Apostilas/Controle\%20tecnologico\%20basico\%20do\%20concreto.pdf

2. Associação Brasileira de Normas Técnicas. NBR NM 67: Concreto. Determinação da consistência pelo abatimento do tronco de cone. Rio de Janeiro (RJ): ABNT; 1998.

3. Associação Brasileira de Normas Técnicas. NBR 5739: Concreto - Ensaio de compressão de corposde-prova cilíndricos. Rio de Janeiro (RJ): ABNT; 2007.

4. Associação Brasileira de Normas Técnicas. NBR 7211: Agregado para Concreto. Rio de Janeiro (RJ): ABNT; 2005.

5. Associação Brasileira de Normas Técnicas. NBR 7222: Argamassa e concreto - Determinação da resistência à tração por compressão diametral de corpos-de-prova cilíndricos. Rio de Janeiro (RJ): ABNT; 1994.

6. Associação Brasileira de Normas Técnicas. NBR 11578: Cimento Portland Composto. Rio de Janeiro (RJ): ABNT; 1997.

7. Associação Brasileira de Normas Técnicas. NBR 15530: Fibras de aço para concreto - Especificação. Rio de Janeiro (RJ): ABNT; 2007.

8. Bentur A, Mindess S. Fibre Reinforced Cementitious. New York: Elsevier Science Publishers; 1990.

9. Figueiredo AD. Concreto reforçado com fibras [tese]. São Paulo (SP): Universidade de São Paulo; 2011. $248 \mathrm{p}$.

10. Góis FAP. Avaliação Experimental do Comportamento de Concreto Fluido Reforçado com Fibras de Aço: Influência do fator de forma e da fração volumétrica das fibras nas propriedades mecânicas do concreto [dissertação]. Maceió (AL): Universidade Federal de Alagoas; 2010. 156 p.

11. Mehta PK, Monteiro PJM. Concreto: microestrutura, propriedades e materiais. São Paulo: Instituto Brasileiro do Concreto; 2008.

12. Pujadas P. Caracterización y diseño del hormigón reforzado con fibras plásticas [tese]. Cataluna (Barcelona): Universitat Politècnica de Catalunya; 2013. 